\title{
El Gobierno Electrónico y la Implementación de las TIC para Brindar Nuevos Canales de Comunicación
}

\author{
Rocío A. Rodríguez, Pablo M. Vera, Isabel B. Marko, Claudia G. Alderete, Anabella G. Conca \\ GIDFIS (Grupo de Investigación, Desarrollo y Formación en Innovación de Software) \\ Departamento de Ingeniería e Investigaciones Tecnológicas \\ Universidad Nacional de La Matanza \\ San Justo, Buenos Aires, Argentina \\ gidfis@ing.unlam.edu.ar
}

\begin{abstract}
Resumen - El presente artículo expone la situación actual del gobierno electrónico desde un punto de vista meramente tecnológico, en función del grado de implementación de la tecnología en beneficio de los ciudadanos. No obstante este es un tema amplio que puede ser analizado desde distintas perspectivas y si bien no es el objetivo del artículo realizar una evaluación enfocada a lo social, cultural y político, tampoco nuestro enfoque puede estar completamente aislado de estas cuestiones no menores, que influyen en el momento de tomar decisiones de construir o no, determinada solución tecnológica. En este artículo se plantearan las bases del gobierno electrónico, la evolución histórica y las fases de implementación, para finalmente presentar la situación actual basada en un relevamiento recientemente realizado que dejará en evidencia que aún queda mucho por hacer en materia de gobierno electrónico.
\end{abstract}

Palabras Clave - Gobierno Electrónico, e-government, tecnología, dispositivos móviles, sitios web, sitios web móviles, diseño adaptativo, responsive design.

\section{GOBIERNO ELECTRÓNICO}

La inclusión masiva de las TIC (tecnologías de la información y comunicación) es un hecho significativo.

"Es importante dar una mirada al grado de aprovechamiento de las potencialidades de las TIC y en general de la comunicación digital, para mitigar las problemáticas humanitarias que presentan las sociedades actuales" [1].

El desarrollo del potencial de las TIC, en múltiples sectores de la sociedad se ve impulsado desde dos aspectos: "la generalización del uso de las TIC desde los hogares y la emergencia de nuevas oportunidades tecnológicas por parte de los gobiernos" [2].

El uso masivo de internet desde computadoras de escritorio y más aún desde dispositivos móviles ha cambiado radicalmente la forma en que se comunican las personas. La alta penetración de la telefonía celular, en donde hay mayor cantidad de líneas activas que de habitantes [3], trae consigo la necesidad de plantear nuevos escenarios de comunicación. Esto impacta también en la forma en que el Gobierno se actualiza incorporando las TIC a sus procesos y permite la modernización de la Gestión Pública.

Existen distintos espacios de comunicación virtual, los cuales son tratados por diversos autores [4], [5], [6], [7]): Gobierno-Gobierno (G2G), Gobierno-Empleados (G2E), Gobierno-Empresas (G2B), Gobierno-Ciudadanos (G2C), Gobierno-Visitantes (G2V). En la figura 1 se presentan dichos espacios de comunicación englobados en tres niveles. Puede evidenciarse que existen relaciones internas (G2E, G2G) y relaciones externas (G2C, G2B, G2V). Generando 2 dimensiones dadas por "el uso de TIC, hacia adentro de la administración pública (Back Office) o hacia los otros actores que, de una u otra manera, se relacionan con ella a través de la pantalla (Front Office)" [8].

Estos espacios plantean la forma en que se comunica el Gobierno, entre ellas este artículo se focaliza a G2C (GobiernoCiudadanos). Lo que ha dado origen a definiciones puntuales del Gobierno Electrónico planificadas para este espacio comunicacional: "La Gobernabilidad Electrónica (eGovernance) se refiere al uso de las tecnologías de la información y la comunicación por parte del sector público con el objetivo de mejorar el suministro de información y el servicio proporcionado. De esta manera, se trata de estimular la participación ciudadana en el proceso de toma de decisiones, haciendo que el gobierno sea más responsable, transparente y eficaz" [9].

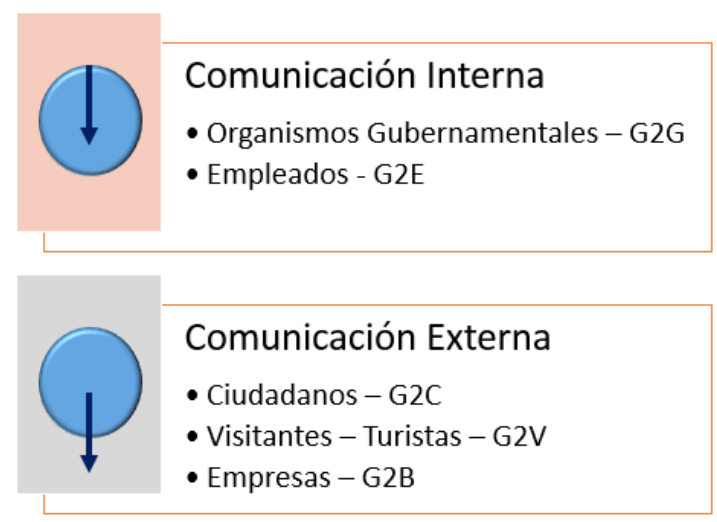

Fig. 1. Espacios de comunicación virtual

"El Gobierno Electrónico entrega beneficios directos a la comunidad en general, tales como la eliminación de las barreras de tiempo y espacio, la facilidad en las comunicaciones, el acceso igualitario a la información, el aumento del intercambio colaborativo entre distintas reparticiones, el incremento en la producción de bienes y servicios de valor agregado; en suma, una mayor calidad de vida de la ciudadanía. Por su parte, la utilización de estas tecnologías en la gestión pública puede traer grandes beneficios, pues constituyen pilares fundamentales para la modernización y eficacia del Estado, ayudan al control interno y externo aportando transparencia al sector público, disminuye costos del sector público al compartir recursos, ayuda a la descentralización acercando el Gobierno a los ciudadanos y 
facilita la participación ciudadana en los procesos de tomas de decisiones, entre otros" [10].

Los países han considerado de importancia implementar las tecnologías para la modernización del estado y ofrecer mediante diversos canales electrónicos nuevas alternativas a los ciudadanos para poder acceder a información, realizar tramitaciones, enviar consultas, etc. En Argentina en el año 2005 [11] se establece el Plan Nacional de Gobierno Electrónico (Decreto 378/2005), iniciativa que no es aislada sino que es compartido el interés por distintos países que comienzan a establecer bases para evaluar el grado de implementación del gobierno electrónico o promover buenas prácticas. Una muestra de ello es la Conferencia Iberoamericana de Ministros de Administración Pública y Reforma del Estado, en la cual se genera la redacción de Cartas Iberoamericanas. La Carta Iberoamericana de Gobierno Electrónico, en el 2007, fue consensuada por 21 países (Argentina, Bolivia, Brasil, Chile, Colombia, Costa Rica, Cuba, Ecuador, El Salvador, España, Guatemala, Honduras, México, Nicaragua, Panamá, Paraguay, Perú, Portugal, República Dominicana, Uruguay, Venezuela) [12] que acuerdan las bases fundamentales para su implementación del Gobierno Electrónico. "La implantación del Gobierno Electrónico comporta el reconocimiento por parte de los Estados Iberoamericanos del derecho de los ciudadanos a relacionarse electrónicamente con sus Gobiernos y Administraciones Públicas. Lo que supone que las Administraciones estén interrelacionadas entre sí a fin de simplificar los procedimientos" [12]. Siendo un instrumento muy significativo. En ella se establecen 7 principios del Gobierno Electrónico:

- Igualdad: Todo lo que puede hacerse con medios electrónicos también seguirá realizándose en las Administraciones Públicas por medios no electrónicos.

- Legalidad: Las garantías previstas en los medios tradicionales de relación con el Gobierno y Administraciones Públicas, se mantendrán idénticas en los medios tecnológicos.

- Responsabilidad: La forma en que respondan por sus actos por medios electrónicos deberá ser la misma que por medios tradicionales. No deben existir cláusulas o leyendas de "irresponsabilidad".

- Conservación: Las comunicaciones y documentos electrónicos se conservan en las mismas condiciones que por los medios tradicionales.

- Transparencia y Accesibilidad: La información y el conocimiento de los servicios electrónicos tendrá un lenguaje comprensible según el perfil del destinatario.

- Proporcionalidad: Seguridad adecuada.

- Adecuación Tecnológica: Las administraciones elegirán las tecnologías más adecuadas para satisfacer sus necesidades (computadora, la televisión digital, los mensajes SMS en teléfonos celulares, etc ...).

En el 2008, surge una Carta Iberoamericana de Calidad en la Gestión Pública, en la cual se hace alusión al gobierno electrónico "De conformidad con la "Carta Iberoamericana de Gobierno Electrónico", la Administración Pública buscará la optimización de las tecnologías de la información y la comunicación, para la mejora y acercamiento del servicio a las necesidades ciudadanas, así como de la propia gestión pública, mejora de la atención, trámites en línea, participación y transparencia. Para ello, tratará de facilitar múltiples modalidades de acceso y realización de trámites, así como el intercambio documental y de información en ambos sentidos Administración-ciudadano" [13].

En el 2009, surge una Carta Iberoamericana de Participación Ciudadana en la Gestión Pública [14], la cual hace referencia a la de Gobierno Electrónico.

Estas iniciativas demuestran un interés marcado por establecer el uso de las TIC en la administración pública. En base a lo plasmado se esperaría que luego de 10 años el grado de implementación y madurez de los servicios y tecnologías aplicadas al gobierno electrónico sea inminente. No obstante existen algunas limitaciones a las que aún se enfrentan los organismos, entre ellos se destaca:

- Resulta complejo realizar un cambio tan radical en muchas instituciones cuyas líneas de acción están basadas en pasos estructurados mediante documentación extensa y rudimentaria.

- Existe aún brecha tecnológica entre los nativos digitales y las generaciones que son inmigrantes en los temas relacionados con la tecnología, que es difícil de subsanar. Esta brecha no sólo está presente en los ciudadanos que serán los receptores de la implantación de soluciones tecnológicas, sino también en quienes ocupan puestos jerárquicos y deben tomar la decisión si invertir o no en tecnología.

- Los presupuestos ajustados y las entidades públicas no siempre se destinan parte de la partida presupuestaria en la construcción de soluciones tecnológicas y esto hace que el personal en algunos casos sin capacitación alguna, haga esfuerzos por llevar a cabo una solución puntual, sin tener tampoco tiempos asignados para esta tarea concreta.

En base a estas limitantes es posible abordar algunas reflexiones, (1) Es necesario aceptar que la forma en que la sociedad se comunica ha cambiado y que las TIC se han instalado desde los más profundo de la sociedad. No obstante todo cambio debe traer consigo un beneficio y poder modernizar a la administración pública, reduciendo tiempos y facilitando procedimientos que son engorrosos; es altamente importante. Pero los integrantes de estas administraciones deben estar preparados para este cambio. (2) Si bien es cierto que existen importantes esfuerzos desde el gobierno y el ámbito académico, aún quedan ciudadanos que no están en condiciones por cuestiones socio-económicas de utilizar la tecnología. Si bien se avanza incorporando las TIC en las clases y acercando este conocimiento a adultos mayores; no debe esperarse que todos sean capaces de realizar gestiones a través de medios tecnológicos. Es por ello que cada nuevo canal debe sumarse a los ya existentes. (3) Los presupuestos suelen ser ajustados y no siempre se destina partida presupuestaria a cuestiones vinculadas con la tecnología, esto redunda entonces en problemas de baja implementación o implementación deficiente en muchos casos.

"Implementar un Gobierno Electrónico implica, entre otras actividades, replantear, agregar y/o eliminar procesos, definir políticas de calidad y seguridad, analizar los procesos de negocio en cada uno de los servicios públicos, todo esto en vías de lograr la integración e interoperabilidad de estos servicios. Las soluciones tecnológicas que ayudan a esta tarea deben ser capaces de responder de forma óptima a las necesidades de integración derivadas de la existencia de entornos heterogéneos, tanto en aplicaciones como en sistemas. Sin embargo, debe considerarse que medir desempeño y avance de las políticas de Gobierno Electrónico trae consigo no sólo aspectos técnicos (como la tasa de penetración de las TIC), sino que es necesario también considerar aspectos culturales, 
organizacionales, políticos, sociales, entre otros. La clave está, desde luego, en la implementación de programas y políticas más eficaces" [10].

Actualmente el Gobierno Electrónico mantiene un alto grado de importancia para la comunidad, que puede analizarse en forma creciente en la web por medio de la tendencia del tratamiento de la temática (esto puede observarse en la figura 2 , generada por medio de google trends).

No hay duda alguna que el gobierno electrónico presenta nuevos desafíos, surgiendo nuevos espacios de comunicación y nuevas herramientas que brindan a los ciudadanos alternativas de acción que se suman a las ya existentes.

\section{FASES DE IMPLEMENTACIÓN}

El grado de implementación del gobierno electrónico puede analizarse mediante 5 fases, las cuales se presentan en la figura 3. Estas fases implican un progreso incremental en cuanto a los objetivos alcanzados, es decir que para conseguir alcanzar la fase de integración total deberían estar incorporados los mecanismos necesarios para la fase transaccional [15] y por consecuencia, todas las anteriores.

Cabe destacar que es posible realizar distintas acciones que incorporen tecnología a beneficio de los ciudadanos. Se tomará en esta sección a modo de ejemplo los Sitios Web Oficiales para ejemplificar cada una de las fases de implementación.

- Emergente, la decisión está tomada y se entiende la importancia del gobierno electrónico pero aún se cuenta con un sitio web oficial básico en el que se muestra información estática, que no requiere actualización como por ejemplo el objetivo de la entidad y un listado de trámites que se deben hacer personalmente en la institución, normalmente no se cuenta con noticias o estas se encuentran desactualizadas dejando en evidencia que estos sitios no tienen actualización frecuente. Aún hay municipios que se encuentran en esta etapa.

- Ampliado, se aumentan las posibilidades dándole a la ciudadanía la facultad de contactarse con el organismo, los sitios web tienen valor agregado que incluyen la posibilidad de participación ciudadana, no son meros repositorios de información.

- Iterativo, aquí la atención se centra en la participación ciudadana, en donde los municipios no sólo publican sus presupuestos sino también existe el concepto de presupuesto participativo.
- Transaccional, existe la posibilidad de realizar gestiones complejas de una forma segura y sencilla. Muchas instituciones públicas han comprendido la importancia de ofrecer trámites en línea y lo han implementado de forma exitosa.

- Integración total, esto abarca conceptos tales como interoperabilidad de las soluciones ofrecidas, poder unificar formas de realizar tramitaciones. Esto beneficiará en que no todas las instituciones tengan que construir sus propias soluciones y puedan consumir servicios ofrecidos a nivel global. El problema radica en que no todos los servidores utilizan la misma tecnología, no todos los municipios tienen el mismo equipamiento y en muchos casos se desconoce los recursos de software existentes que podrían ser compartidos. En esta fase deberá analizarse como se puede transmitir datos entre organismos evitando de este modo duplicidad de la información y la mejora de la actualización de la misma.

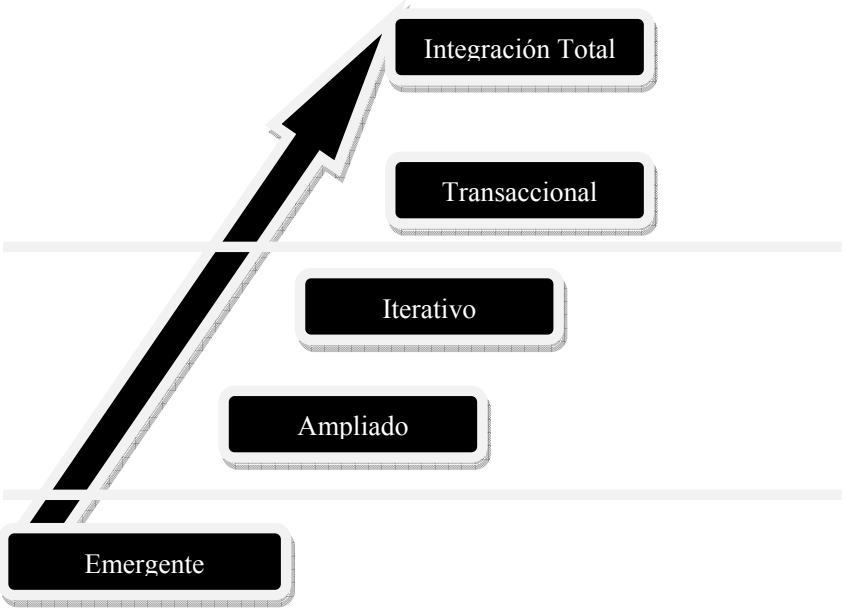

Fig. 3. Fases de Implementación

"Esto implica una evolución no sólo tecnológica sino también cultural, ya que la incorporación de las TIC en la gestión pública impacta tanto a los organismos públicos como a la sociedad en su conjunto" [10]. "La apropiación por parte de los habitantes se desarrolla en un espacio de tensión entre las resistencias y la aceptación entusiasta. La atracción y el temor coexisten, aún en los propios usuarios" [16].

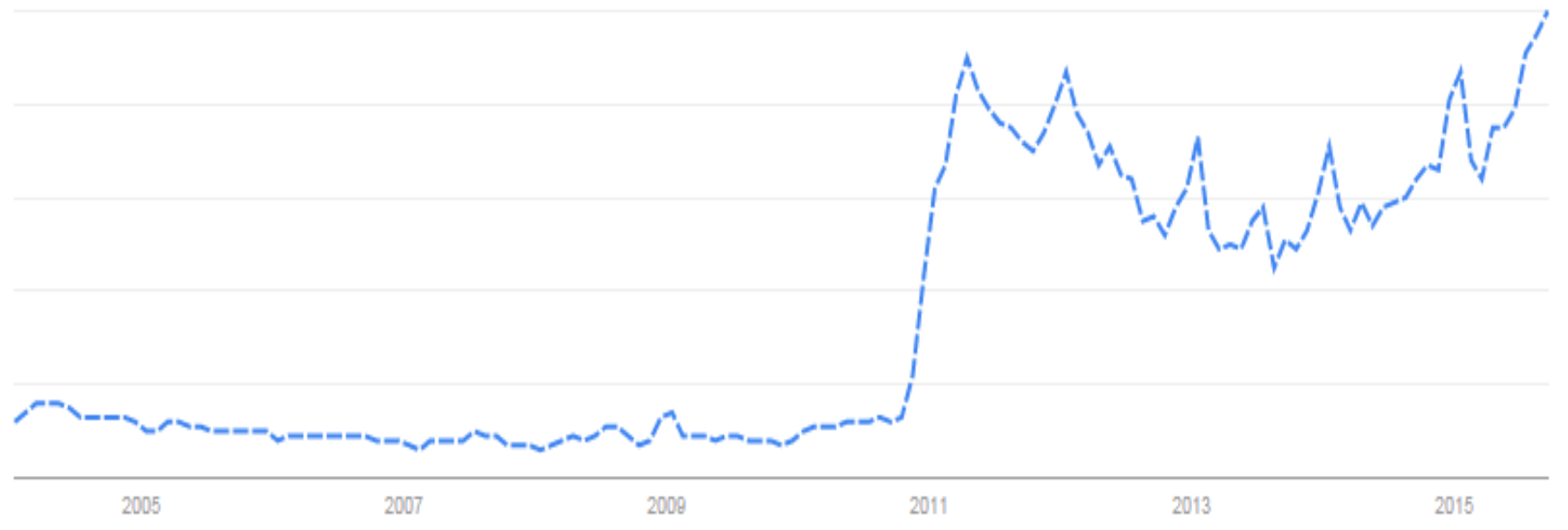

Fig. 2. Interés - Término e-government en la web 


\section{TECNOLOGÍAS IMPLEMENTADAS}

El Gobierno Electrónico requiere la implementación de nuevos canales tecnológicos que se suman a los medios tradicionales existentes. Es por ello que resulta indispensable analizar el uso que se le dará a cada canal y como ofrecer en él los recursos dirigidos al ciudadano de forma exitosa.

"Vivimos en tiempos en que se presta una atención extraordinaria a una serie de dispositivos que ayudan al intercambio de información y la comunicación entre las personas. Cada día más habitantes del planeta parecieran necesitar de estos aparatos. Casi en todo orden de cosas el acceso a estos dispositivos parece esencial, ya no sólo para permitir la interacción a distancia entre individuos, sino que también para facilitar el comercio, la ciencia, el entretenimiento, la educación, y un sinnúmero de actividades relacionadas con la vida moderna..." [17].

Dada la alta inserción de dispositivos móviles, es importante considerar que estos dispositivos constituyen un nuevo canal de comunicación, para mejorar el espacio virtual G2C. Producto de esto ha surgido el m-government, el cual puede ser definido "Como estrategia y su implementación involucrando la utilización de toda clase de tecnología, móvil e inalámbrica, servicios y aplicaciones y dispositivos para mejorar los beneficios de las partes involucradas e-government incluyendo a los ciudadanos, las empresas y todas las unidades gubernamentales" [18]. Una iniciativa de interés es considerar a los SMS como un recurso simple de comunicación [19].

En base al informe del 2014 realizado por las Naciones Unidas [20], se construye la figura 4 la cual muestra la cantidad de portales nacionales que incluyen al SMS como canal de comunicación entre el Gobierno y los Ciudadanos, para el año 2014 son 32 los países pudiendo notarse que la cantidad va en aumento.

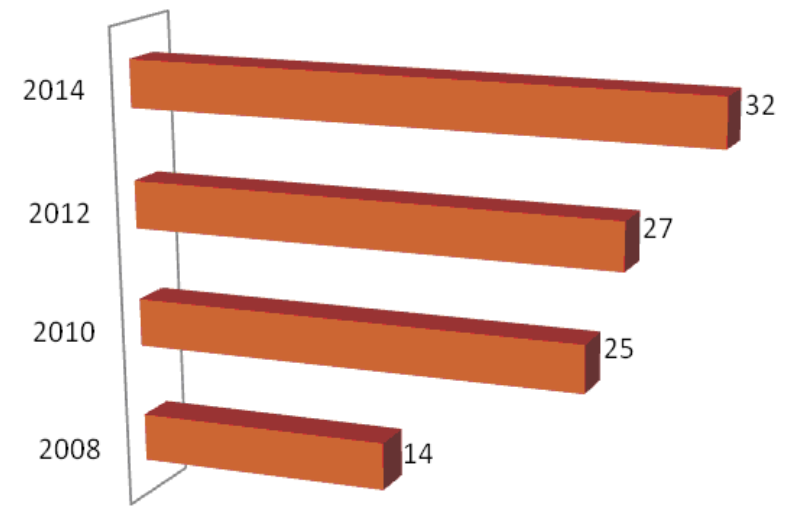

Fig. 4. Cantidad países por año que en sus Portales Nacionales ofrecen los SMS como canal de Comunicación G2C

"La ventaja de utilizar estos canales es que la mayor parte de la población tiene a su alcance un teléfono móvil; (...). De forma que sería un canal fácil de utilizar y rápido para enviar y recibir comunicaciones" [21].

Existen algunas iniciativas de implementación de los SMS para brindar distintos servicios los cuales pueden observarse en forma resumida en la tabla I.

La tabla 1 muestra que existen diversas iniciativas de implementación de m-servicios utilizando al SMS a modo de instrumento. Como puede observarse en Argentina existen diferentes servicios implementados.

Cabe destacar que algunos municipios por ejemplo en España implementan el WhatsApp como medio de

comunicación, que no implica gasto alguno para el municipio ni para el ciudadano.

TABLA I. SERVICIOS OFRECIDOS POR SMS

\begin{tabular}{|c|c|c|}
\hline $\begin{array}{l}\text { Tipo de } \\
\text { Servicio }\end{array}$ & Descripción & $\begin{array}{l}\text { Ejemplos de lugares de } \\
\text { Implementación }\end{array}$ \\
\hline $\begin{array}{l}\text { Estacionami } \\
\text { ento Medido }\end{array}$ & $\begin{array}{l}\text { La tarifa se descuenta del } \\
\text { crédito del celular ó bien se } \\
\text { carga crédito específicamente } \\
\text { para este fin para una línea de } \\
\text { abonado. }\end{array}$ & $\begin{array}{l}\text { - Argentina } \\
\text { - Australia } \\
\text { - Bélgica } \\
\text { - Brasil } \\
\text { - Emiratos Árabes } \\
\text { - Estados Unidos } \\
\text { - Estonia } \\
\text { - Holanda }\end{array}$ \\
\hline \multirow{10}{*}{$\begin{array}{l}\text { Alertas } \\
\text { y Avisos } \\
\text { Suscripción } \\
\text { a un servicio, } \\
\text { de temas de } \\
\text { interés para } \\
\text { el usuario }\end{array}$} & $\begin{array}{l}\text { Emergencias Naturales } \\
\text { (Granizo, tormentas, terremotos, } \\
\text { inundaciones, tsunamis, etc.). }\end{array}$ & $\begin{array}{l}\text { - Chile } \\
\text { - Estados Unidos }\end{array}$ \\
\hline & $\begin{array}{l}\text { Emergencias sociales: } \\
\text { (incendios, posibles ataques } \\
\text { terroristas, delincuentes } \\
\text { fugados, personas extraviadas, } \\
\text { etc. }\end{array}$ & $\begin{array}{ll}\text { - } & \text { Australia } \\
& \text { (Victoria) } \\
\text { - } & \text { Estados Unidos } \\
& \text { (New York) } \\
\text { - } & \text { México } \\
\text { - } & \text { Reino Unido } \\
& \text { (Londres) }\end{array}$ \\
\hline & $\begin{array}{l}\text { Fechas de vencimiento de } \\
\text { diversas tasas o servicios }\end{array}$ & $\begin{array}{ll}\text { - } & \text { España } \\
& \text { (Barcelona) } \\
\text { - } & \text { México } \\
& \text { (Zacatecas) } \\
\text { - } & \text { Singapore }\end{array}$ \\
\hline & Congestión de transito & $\begin{array}{ll}\text { - } & \text { España } \\
& \text { (Cataluña) } \\
\text { - } & \text { Estados Unidos } \\
& \text { (New York) } \\
\text { - } & \text { Reino Unido } \\
& \text { (Londres) } \\
\end{array}$ \\
\hline & $\begin{array}{l}\text { Vacantes de Empleo, empresas } \\
\text { registradas con el gobierno, } \\
\text { información de posibles } \\
\text { entrevistas laborales con } 24 \text { hs } \\
\text { de antelación }\end{array}$ & - Brasil \\
\hline & $\begin{array}{l}\text { Atención Médica: El sistema } \\
\text { avisa por SMS cuando el } \\
\text { médico está atendiendo al } \\
\text { paciente anterior, para que los } \\
\text { pacientes puedan esperar en } \\
\text { otros lugares, no sólo en la sala } \\
\text { de espera }\end{array}$ & $\begin{array}{l}\text { - Venezuela } \\
\text { Sucre }\end{array}$ \\
\hline & $\begin{array}{l}\text { Discapacitados auditivos: Povee } \\
\text { alertas, consejos, instrucciones } \\
\text { y notificaciones por medio de } \\
\text { texto que el usuario pueda leer. }\end{array}$ & $\begin{array}{ll}\text { - } & \text { España } \\
& \text { Valencia } \\
\text { - } & \text { Reino Unido } \\
& \text { East Sussex } \\
\end{array}$ \\
\hline & $\begin{array}{l}\text { Plagas de cultivos: Servicio para } \\
\text { agricultores, se indica } \\
\text { posibilidad de plagas. }\end{array}$ & $\begin{array}{l}\text { - Argentina } \\
\text { La Pampa- INTA }\end{array}$ \\
\hline & $\begin{array}{l}\text { Planes Sociales: Información de } \\
\text { la fecha en que va a poder } \\
\text { cobrar el plan social. }\end{array}$ & $\begin{array}{ll}\text { - } & \text { Argentina } \\
& \text { ANSES } \\
\text { - } & \text { Colombia }\end{array}$ \\
\hline & $\begin{array}{l}\text { Recomendaciones, consejos } \\
\text { útiles sobre un tema especifico, } \\
\text { esto se realiza previa } \\
\text { subscripción de un ciudadano al } \\
\text { servicio. Por ejemplo: para } \\
\text { evitar accidentes de tránsito. }\end{array}$ & $\begin{array}{l}\text { - Argentina } \\
\text { - Ministerio del Interior }\end{array}$ \\
\hline \multirow[t]{4}{*}{$\begin{array}{l}\text { Consultas } \\
\text { Enviadas por } \\
\text { el ciudadano }\end{array}$} & Deudas de tasas o impuestos & $\begin{array}{l}\text { - } \text { Argentina } \\
\text { AFIP -ARBA } \\
\text { - Costa Rica } \\
\text { Sarapiquí } \\
\end{array}$ \\
\hline & Padrón electoral & $\begin{array}{l}\text { - } \text { Argentina } \\
\text { Ministerio del Interior } \\
\text { - España } \\
\text { Barcelona } \\
\text { - } \text { Venezuela }\end{array}$ \\
\hline & Seguimiento de un trámite & $\begin{array}{l}\text { - } \text { Costa Rica } \\
\text { Sarapiquí } \\
\end{array}$ \\
\hline & $\begin{array}{l}\text { Chequeo de situación de un } \\
\text { vehículo usado }\end{array}$ & - Nueva Zelanda \\
\hline
\end{tabular}




\begin{tabular}{|c|c|c|}
\hline $\begin{array}{l}\text { Tipo de } \\
\text { Servicio }\end{array}$ & Descripción & $\begin{array}{l}\text { Ejemplos de lugares de } \\
\text { Implementación }\end{array}$ \\
\hline & $\begin{array}{l}\text { Conocer la disponibilidad de } \\
\text { anclajes y bicicletas }\end{array}$ & $\begin{array}{l}\text { - España } \\
\text { Barcelona }\end{array}$ \\
\hline & $\begin{array}{l}\text { Puntos de WI-FI en Barcelona } \\
\text { en el código postal de interés }\end{array}$ & $\begin{array}{l}\text { - España } \\
\text { - Barcelona }\end{array}$ \\
\hline & $\begin{array}{l}\text { Información de la grúa municipal } \\
\text { (ubicación del vehículo, costo de } \\
\text { la tasa, tramitación) }\end{array}$ & $\begin{array}{l}\text { - España } \\
\text { (Barcelona) }\end{array}$ \\
\hline Encuestas & $\begin{array}{l}\text { Es posible consultar a la } \\
\text { ciudadanía sobre algún tópico o } \\
\text { preferencia específica. Los } \\
\text { ciudadanos con un simple } \\
\text { mensaje de texto pueden } \\
\text { contestar un determinado } \\
\text { interrogante. }\end{array}$ & $\begin{array}{l}\text { - } \quad \text { Australia } \\
\text { - España (Guadalajara) }\end{array}$ \\
\hline Denuncias & $\begin{array}{l}\text { Quejas de: servicios de } \\
\text { gobierno, proyectos, } \\
\text { funcionarios... }\end{array}$ & - México \\
\hline $\begin{array}{l}\text { Reserva de } \\
\text { Turnos en } \\
\text { hospitales }\end{array}$ & $\begin{array}{l}\text { Permite solicitar turnos vía SMS } \\
\text { ingresando la especialidad } \\
\text { médica y/o la fecha requerida. } \\
\text { Se responde con otro SMS que } \\
\text { otorga un turno posible que el } \\
\text { paciente deberá aceptar o } \\
\text { rechazar, por medio de otro } \\
\text { SMS. }\end{array}$ & - Argentina Misiones \\
\hline Concursos & $\begin{array}{l}\text { Una nueva forma de mejorar la } \\
\text { comunicación entre la sociedad } \\
\text { y los organismos permitiendo } \\
\text { incluir a los más jóvenes, por } \\
\text { ejemplo a través de concursos } \\
\text { sobre frases creativas, lemas, } \\
\text { slogans municipales, etc. }\end{array}$ & $\begin{array}{l}\text { - España } \\
\text { Andalucía }\end{array}$ \\
\hline $\begin{array}{l}\text { Notificacion } \\
\text { es } \\
\text { Certificadas } \\
\text { /Resolucion } \\
\text { es de } \\
\text { Gobierno }\end{array}$ & $\begin{array}{l}\text { Las entidades gubernamentales } \\
\text { pueden envían notificaciones } \\
\text { certificadas (con valor legal), e } \\
\text { informar sobre resoluciones del } \\
\text { organismo. }\end{array}$ & $\begin{array}{l}\text { - España } \\
\text { Lleida - Salou }\end{array}$ \\
\hline $\begin{array}{l}\text { Prevención } \\
\text { y denuncia } \\
\text { de delitos }\end{array}$ & $\begin{array}{l}\text { Los vecinos de una localidad } \\
\text { pueden enviar SMS con } \\
\text { información sobre delitos } \\
\text { antiguos o actuales, o sobre } \\
\text { situaciones sospechosas que } \\
\text { requieran intervención policial. }\end{array}$ & $\begin{array}{l}\text { - } \text { Argentina } \\
\text { Buenos Aires } \\
\text { - Estados Unidos } \\
\text { Boston y New York } \\
\text { - } \\
\text { Australia } \\
\text { Victoria } \\
\text { - } \\
\text { Reino Unido } \\
\text { East Essex y Londres }\end{array}$ \\
\hline
\end{tabular}

De hecho puede verse en las páginas principales de los sitios web, de diversos ayuntamientos de España, la indicación del servicio de WhatsApp para la atención ciudadana (ver figura 5). En la figura 5 puede notarse en los casos de los municipios A, B y C (los dos primeros correspondientes a España y el tercero a Omán), que se ofrece el WhatsApp con algún gráfico de forma destacada; mientras que en $\mathrm{D}$ que corresponde al municipio de Kolkata en India, no se destaca del resto de información de contacto del sitio web.

Los presentados en la figura 5 son tan sólo algunos ejemplos en los cuales se ha implementado WhatsApp como canal de comunicación.

Pero además de ser un simple canal de comunicación como una línea telefónica pero gratuita; algunos municipios lo comienzan a utilizar con otras funcionalidades como es el caso del ayuntamiento de Algimia d'Alfara (España) que informa a la ciudadanía de cortes de calles por este medio. También los ciudadanos pueden enviar mensajes comunicando sobre incidentes en la vía pública (ver figura 6).

En Argentina diversos municipios han implementado el WhatsApp, como vía de comunicación gratuita, canal de mensajería desde el municipio a los ciudadanos y también para reclamos por parte de la ciudadanía (ver tabla II).

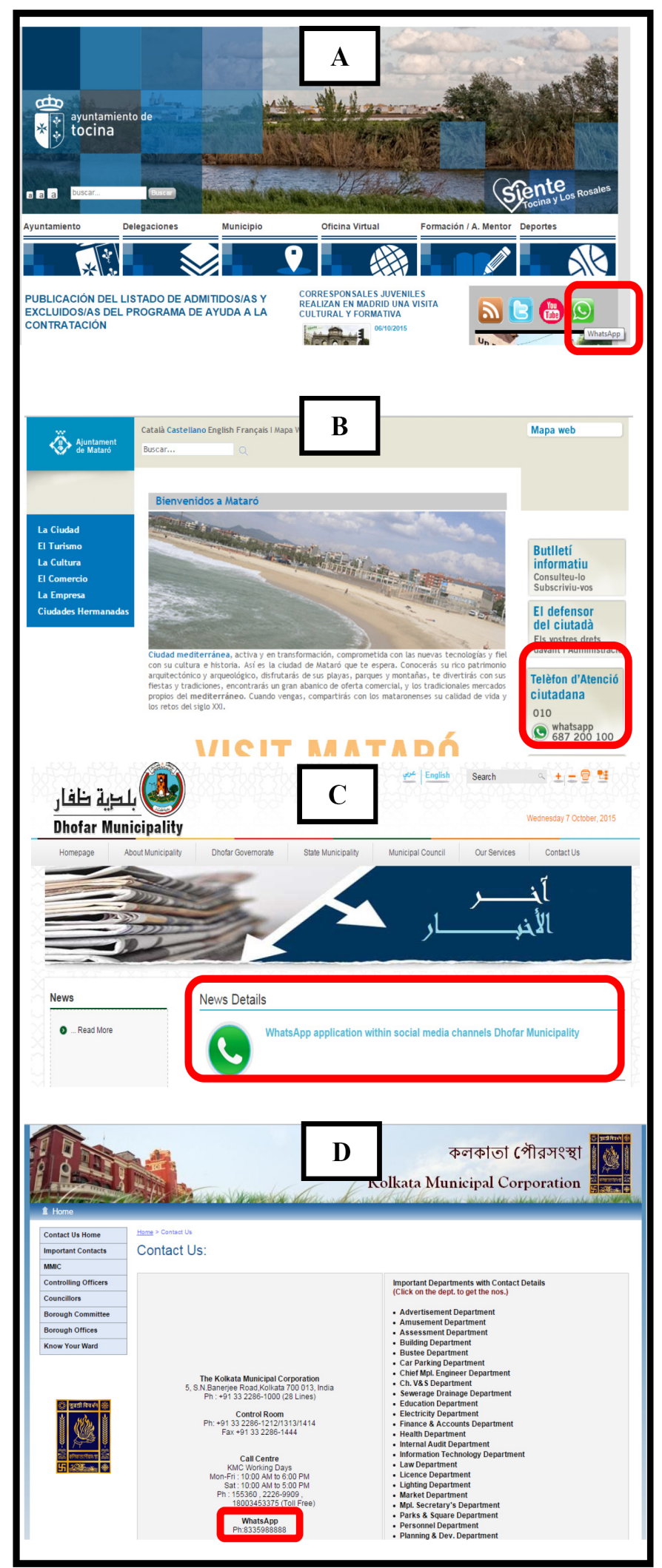

Fig. 5. Capturas de pantalla - Municipios de: A: Tocina, B: Mataró (España) C: Dhofar (Omán) y D: Kolkata (India)

Aún no está implementada la comunicación en dos sentidos Gobierno a Ciudadanos y Ciudadanos a Gobierno, en la mayor parte de los casos, pero es notable como este recurso se transforma en un nuevo canal de comunicación.

Tanto el SMS como el WhatsApp deberían ser canales de comunicación que estén presente en los municipios. 
Algimia d'Alfara

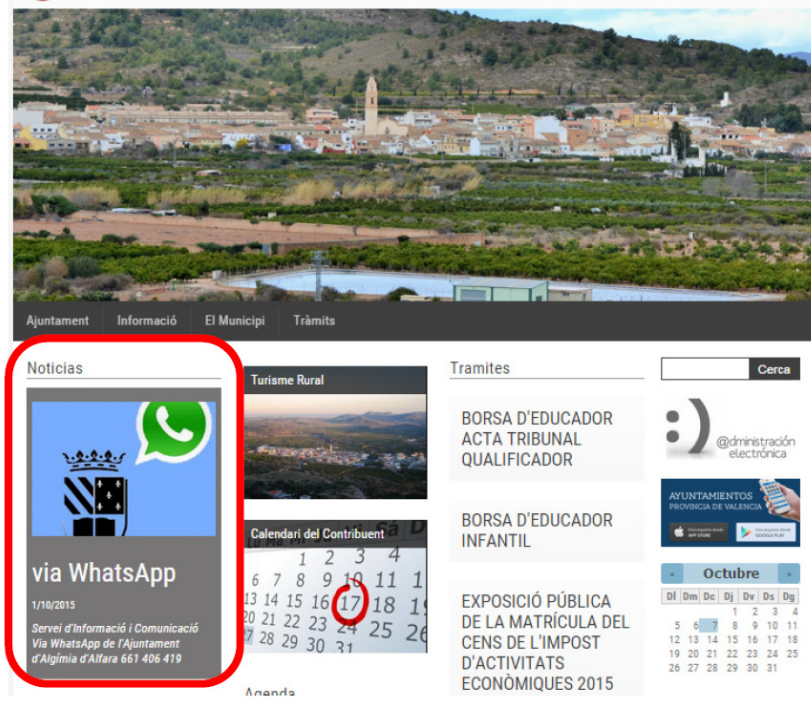

Fig. 6. Captura de pantalla - Ayuntamientos de Algimia d'Alfara (España)

TABLA II. SERVICIOS OFRECIDOS POR WHATSAPP EN ARGENTINA

\begin{tabular}{|c|c|c|}
\hline Tipo de Servicio & Descripción & $\begin{array}{l}\text { Ejemplos de lugares de } \\
\text { Implementación }\end{array}$ \\
\hline $\begin{array}{l}\text { Noticias y Alertas } \\
\text { emitidas por los } \\
\text { municipios }\end{array}$ & $\begin{array}{l}\text { Alerta para retirar la } \\
\text { licencia de Conducir }\end{array}$ & $\begin{array}{l}\text { - Municipalidad de } \\
\text { Hurlingham (Buenos } \\
\text { Aires) }\end{array}$ \\
\hline \multirow{4}{*}{$\begin{array}{l}\text { Envío por parte de } \\
\text { los ciudadanos }\end{array}$} & $\begin{array}{l}\text { Baches en la vía } \\
\text { pública }\end{array}$ & $\begin{array}{l}\text { - Municipalidad de Salta } \\
\text { - Municipalidad de Tres de } \\
\text { Febrero (Buenos Aires) }\end{array}$ \\
\hline & $\begin{array}{l}\text { Problemas en vía } \\
\text { pública (luminarias } \\
\text { rotas o defectuosas, } \\
\text { semáforos que no } \\
\text { funcionan) }\end{array}$ & $\begin{array}{l}\text { - Muncipalidad de Tres de } \\
\text { Febrero (Buenos Aires) }\end{array}$ \\
\hline & $\begin{array}{l}\text { Problemas con la red } \\
\text { cloacal }\end{array}$ & - Municipalidad de Córdoba \\
\hline & Reclamos, Denuncias & $\begin{array}{ll}\text { - } & \text { Municipalidad de Las } \\
& \text { Heras (Santa Cruz) } \\
\text { - } & \text { Municipalidad de Junin } \\
\text { (Buenos Aires) } \\
\text { - } \\
\text { Municipalidad de San } \\
\text { Pedro (Buenos Aires) } \\
\text { - Municipalidad de Tigre } \\
\text { (Buenos Aires) } \\
\end{array}$ \\
\hline
\end{tabular}

\section{RELEVAMIENTO}

Para poder evaluar el grado de implementación de las tecnologías para favorecer el Gobierno Electrónico se efectuó un relevamiento sobre los 135 municipios que conforman a la Provincia de Buenos Aires (el mapa con los nombres de los municipios puede visualizarse en [22]), a lo que se suma la Ciudad Autónoma de Buenos Aires; teniendo de esta forma un cubrimiento geográfico de toda la provincia de Buenos Aires:

Estos municipios de la provincia de Buenos Aires están compuestos por 15.625.084 habitantes (en base a los datos del censo poblacional [23]) a lo que se suma la Ciudad Autónoma de Buenos Aires con 2.890.151 habitantes [24]). Estos 18.515.235 habitantes representan el $46,15 \%$ de la población total de Argentina. Siendo una muestra representativa para evaluar el grado de adopción de las TIC por parte de los gobiernos locales en beneficio de la comunicación G2C.

\section{A. Soluciones Web}

Los sitios web son una forma apropiada de poder ofrecer información a los ciudadanos y esto permite que las entidades públicas tengan presencia. Por lo tanto un sitio web básico permitirá a una entidad pública alcanzar la fase emergente, con un sitio web informativo. Pero deberían los sitios web gubernamentales ofrecer otras opciones entre ellas, poder comunicarse con el organismo, facilitar gestiones, solicitar turnos, etc. En esta era en la que gran parte de la sociedad hace uso de estas tecnologías habitualmente parecería casi trivial exponer la ventaja de tener un sitio web disponible. "Los sitios web Gubernamentales deben ser considerados de manera diferente que otros tipos de sitios web, ya que son el único canal de comunicación oficial de los gobiernos en internet. A través de los sitios gubernamentales, los ciudadanos pueden acceder a la información, interactuar con los funcionarios, formular preguntas, realizar gestiones, etc. Los sitios web gubernamentales son una herramienta muy poderosa para mejorar la comunicación entre los ciudadanos y el gobierno, proporcionando información pública de relevancia pero también incrementando la eficiencia en los servicios públicos"'[25].

Sin embargo en contraposición a lo dicho anteriormente, en Buenos Aires, un 6\% de los municipios no tienen sitio web oficial. No alcanzando la primera fase de implementación "Emergente" que puede comprenderse como tener presencia en la web brindando información al ciudadano.

Actualmente tampoco hay un dominio unificado existiendo diversas alternativas:

- Primeramente hay sitios que son".gov" otros ".gob"

Por ejemplo:

○ http://arrecifes.gob.ar/

○ http://www.azul.gov.ar

Cabe destacar que no es indistinto ".gov" que ".gob" ya que no están reservados ambos dominios y que uno redireccione al otro.

- Anteponen Gran Buenos Aires dentro de su dominio apareciendo ".gba"

Por ejemplo:

○ http://www.sanandresdegiles.gba.gob.ar/

- Algunos incorporan previamente ".mun" para indicar que son Municipios.

Por ejemplo:

○ http://www.rauch.mun.gba.gov.ar/

- http://www.9julio.mun.gba.gov.ar/

Esto deja en evidencia que existe una gran diversidad de URL posibles, sin un estándar.

\section{B. Solución Web Móvil}

Por lo indicado anteriormente, en base a la cantidad de servicios ofrecidos por SMS (ver tabla 1) es posible pensar que los dispositivos móviles son un medio considerado por la Administración Pública. Pero en contraposición con la existencia de servicios basados en los SMS, prácticamente no hay esfuerzos invertidos en la construcción de sitios web móviles. El usuario de un dispositivo móvil tiene algunas características particulares entre ellas, que lo lleva consigo la mayor parte del tiempo. Suele ser el teléfono celular un claro ejemplo de esto y además es de uso normalmente personal. Esto permite poder personalizar las aplicaciones a las necesidades del usuario. Claramente los municipios no han avanzado a nivel aplicación, esta decisión puede ser adecuada si se considera que el desarrollo de una aplicación nativa está condicionado al sistema operativo del usuario. El implementar 
distintas aplicaciones para diversos sistemas operativos y versionados es un costo que no se amerita pudiendo brindar la información y servicios a través de la web. Pero la web tradicional no es apta para estos dispositivos debido a que suelen ser sitios pesados (con sobrecarga de imágenes), contenidos extensos para un usuario en movimiento cuya atención no está exclusivamente en su dispositivo, que requieren de plugins para visualizar contenidos (lo cual no es posible en diversos dispositivos), etc. Es decir, que se debe planificar una solución diferente y adaptada para un usuario móvil. "Es pensar en el móvil como punto de partida, esto obliga a concentrarse en lo esencial de un producto y a hacer foco solo en lo que tiene sentido para este dispositivo" [26].

Es de imaginar, luego de relevar los sitios web de municipios de la provincia de Buenos Aires, que aquellos municipios que no ofrecen una solución web tampoco proveerán una solución móvil. De los municipios restantes, es muy bajo el índice de implementación de una solución web móvil. Tan sólo 5 municipios tienen una solución web móvil (el 3,7\% de la muestra considerada), la cual en algunos casos no se encuentra mantenida, (por ejemplo: mostrando vencimientos para del año 2002 - de hace 13 años), con problemas de visualización de caracteres y otros que dicen estar en construcción. Lo cual deja visible la falta de implementación de las TIC por parte de los gobiernos locales.

Al ingresar una dirección (URL) para acceder a una página web, se envía una petición (request) al servidor donde está alojada dicha página. Luego por cada recurso que incorpore esa página, es decir, imágenes, hojas de estilo, etc. se realiza una nueva petición al servidor para recuperar ese archivo y así poder mostrar la página web con todos los recursos que necesita e incorpora. En la figura 7 se listan la cantidad de request necesaria para cargar la página principal de los sitios web móviles disponibles (Documento, Imágenes, Hojas de Estilo).

Puede observarse en la figura 7, la gran cantidad de request provocada mayormente por la sobrecarga de imágenes de las páginas principales de los sitios móviles.

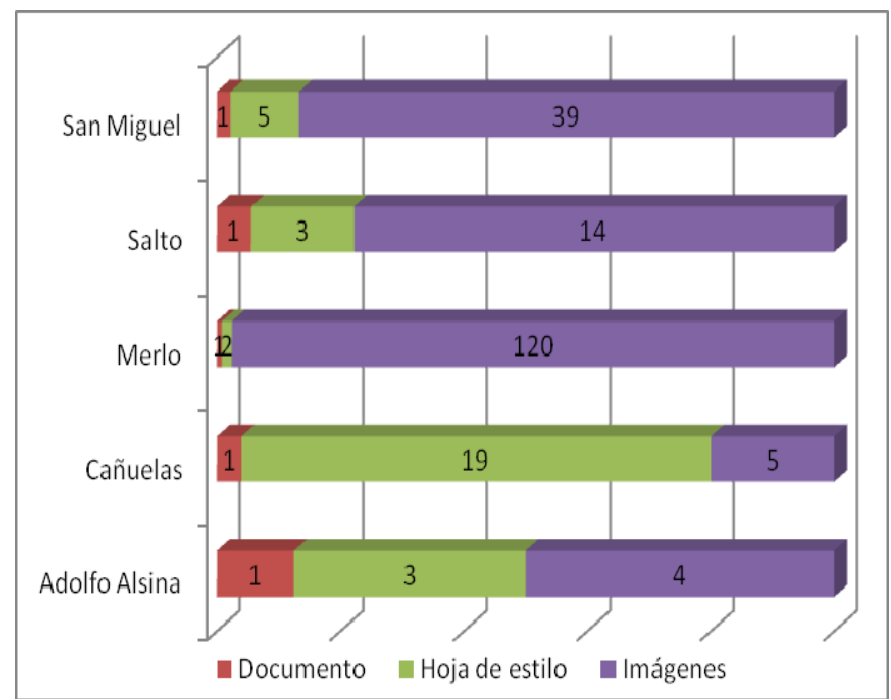

Fig. 7. Cantidad de Request Necesaria para Cargar la Página Principal del Sitio Web Móvil

El peso de estos sitios también es excesivo superando por demás los $20 \mathrm{~KB}$ propuestos por el W3C como uno de los requisitos para asegurar una navegación exitosa para la web desde una amplia gama de dispositivos móviles (ver figura 8).

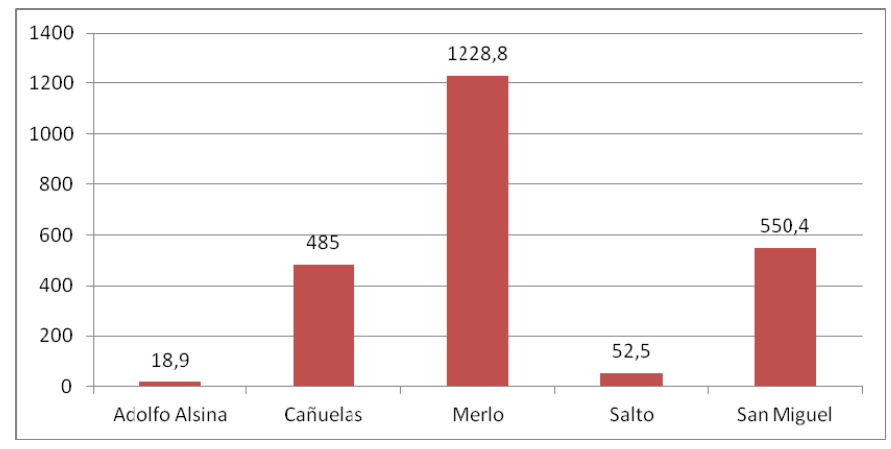

Fig.8. Tamaño en KB total de la página principal de los sitios web móviles

Es importante además, evaluar la diferencia de tamaño entre las soluciones ofrecidas para la web vs la web móvil, medida en KB. En la figura 9 se muestran 2 barras por cada municipio, la barra superior corresponde al sitio web completo y la barra inferior al sitio web móvil.

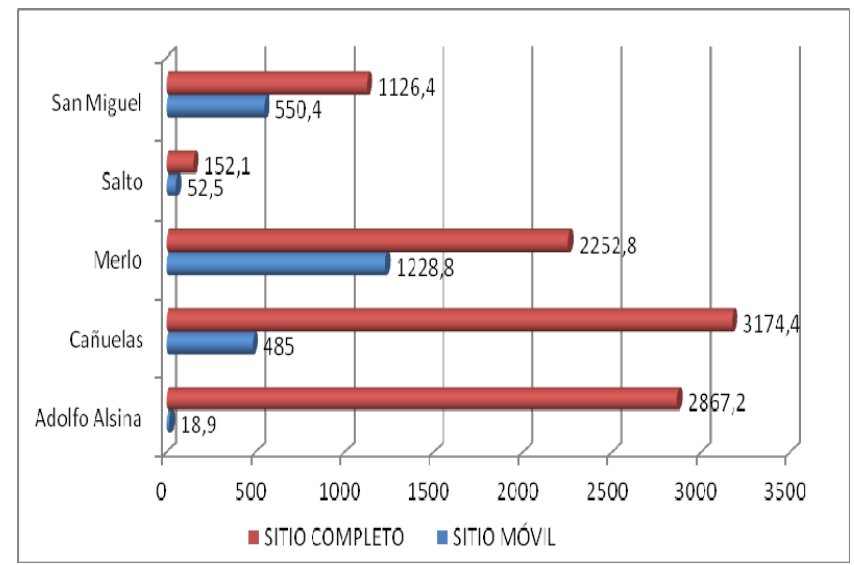

Fig. 9. Diferencia de Tamaño entre los Sitios Web y Móviles

Puede observarse que si bien hay una diferencia marcada entre el tamaño de los sitios web completos y sus versiones móviles, no obstante aún las soluciones web móviles no son livianas como se esperaría para poderse navegar satisfactoriamente desde los distintos dispositivos móviles.

El W3C cuenta con un validador online que permite establecer el porcentaje de cumplimiento de la guía de buenas prácticas para las aplicaciones web móviles [27]. Las buenas prácticas están diseñadas para asegurar una correcta visualización de los sitios web en dispositivos móviles tomando como base unos lineamientos mínimos que deben cumplir los dispositivos mediante los cuales se está accediendo al contenido. Estos lineamientos mínimos fueron diseñados pensando en una web accesible aún desde equipos con bajos recursos, en cuanto a capacidad de procesamiento, dimensiones de pantalla y mecanismos de interacción. Por lo que abarca una amplia gama de dispositivos aún desde dispositivos básicos con acceso a internet como celulares sin un sistema operativo robusto.

En el validador del W3C puede observarse que hay 1 sólo sitio móvil que logra un cumplimiento del 61\% (ver figura 10 ), los 4 restantes dan por resultado el $0 \%$.

Es decir, no sólo son escasos los desarrollos realizados para la web móvil sino que a su vez estos desarrollos presentan grandes deficiencias. Entre ellas la gran cantidad de elementos sobre todo imágenes y el peso excesivo de las mismas, lo que provoca que se retarde el proceso de carga de la página principal. Lo que hace dificultoso acceder a las mismas. 


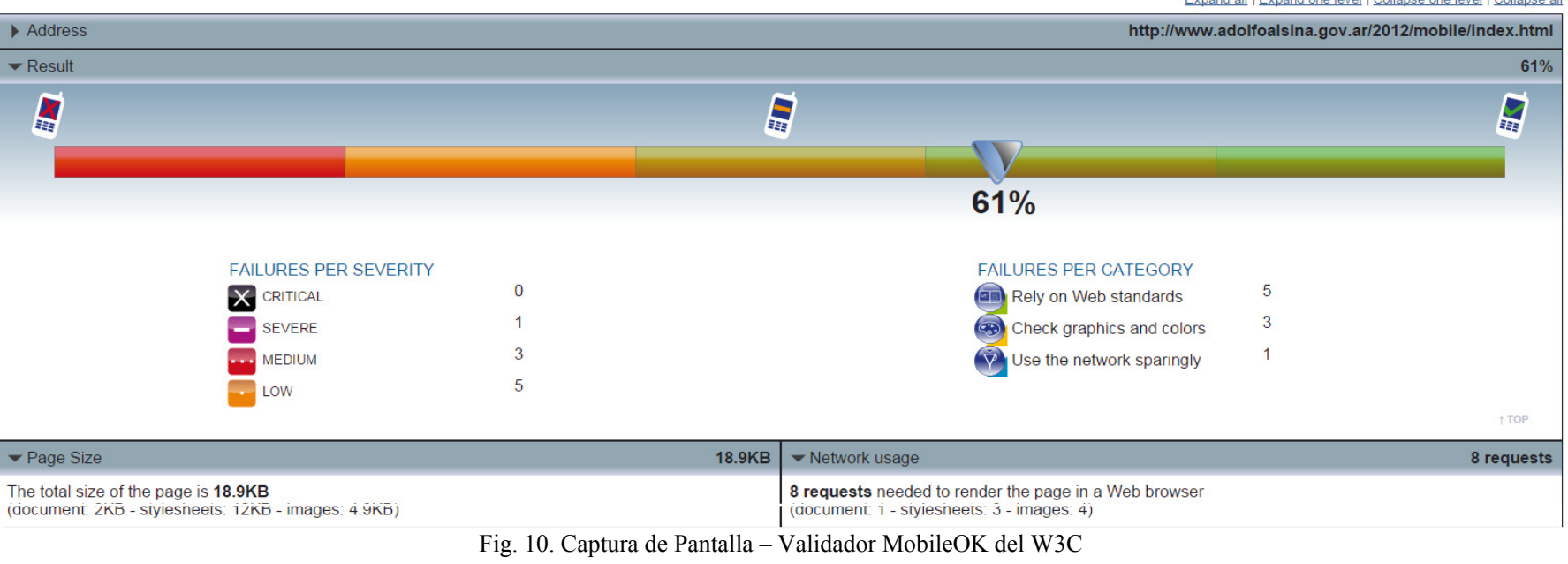

\section{Diseño Adaptativo}

El diseño adaptativo (responsive design) es una buena solución para que un sitio web pueda adaptarse a la visualización en un dispositivo concreto (con una resolución inferior, un tamaño de pantalla más reducido, etc). Permitiendo variar la forma en que se muestra el menú principal, cambiando los mecanismos de navegación, etc. Es un muy buen recurso para aquellos sitios web móviles que podrán ser visualizados de forma más precisa en un teléfono celular ó tablet por ejemplo.

No obstante algunos municipios han optado por aplicar este concepto a sus sitios web tradicionales Es importante comprender que el diseño adaptativo no es una versión diferente del sitio web, sino que es el mismo sitio que se va adaptando y acomodando sus elementos para una mejor visualización en el dispositivo de acceso. Con lo cual no cuenta con todas las ventajas que tendría la construcción de un sitio móvil. Esto trae consigo los mismos inconvenientes comentados previamente en la sección anterior (solución web móvil), pero lógicamente es un recurso adicional para aquellos usuarios que no cuentan con un sitio oficial alternativo de su municipio y desean verlo desde su teléfono celular $\mathrm{u}$ otro dispositivo móvil.

Es decir que el diseño adaptativo no soluciona por si sólo la navegación en los sitios web sino que tan sólo adapta su visualización. El $30,37 \%$ de los sitios web municipales relevados son adaptativos. Esto demuestra que hay un interés incipiente por mejorar los sitios web en beneficio de los usuarios móviles si bien aún falta mucho por hacer. Lo ideal sería ofrecer una versión web tradicional (planificada para PC) y otra solución web móvil (siendo recomendable planificar una solución web móvil para dispositivos básicos y otra para dispositivos avanzados con diseño adaptativo). Del total de los municipios relevados ninguno de ellos cumple con este ideal.

Los sitios web con diseño adaptativo relevados tienen un peso total que va desde los 117,5 KB hasta los 16.076,8 KB; (ver figura 11). Todos ampliamente mayores del peso de 20 $\mathrm{KB}$ propuesto por el $\mathrm{W} 3 \mathrm{C}$, en la figura 11 pueden observarse la cantidad de sitios en cada rango de peso.

Esto deja en evidencia que el diseño adaptativo por sí sólo no es una opción que proporcione sitios web navegables para dispositivos móviles. Por otra parte el diseño adaptativo se logra utilizando CSS Media Queries, lo cual requiere un dispositivo con mayores capacidades de procesamiento. Excluyendo por ende a los dispositivos básicos e intermedios.

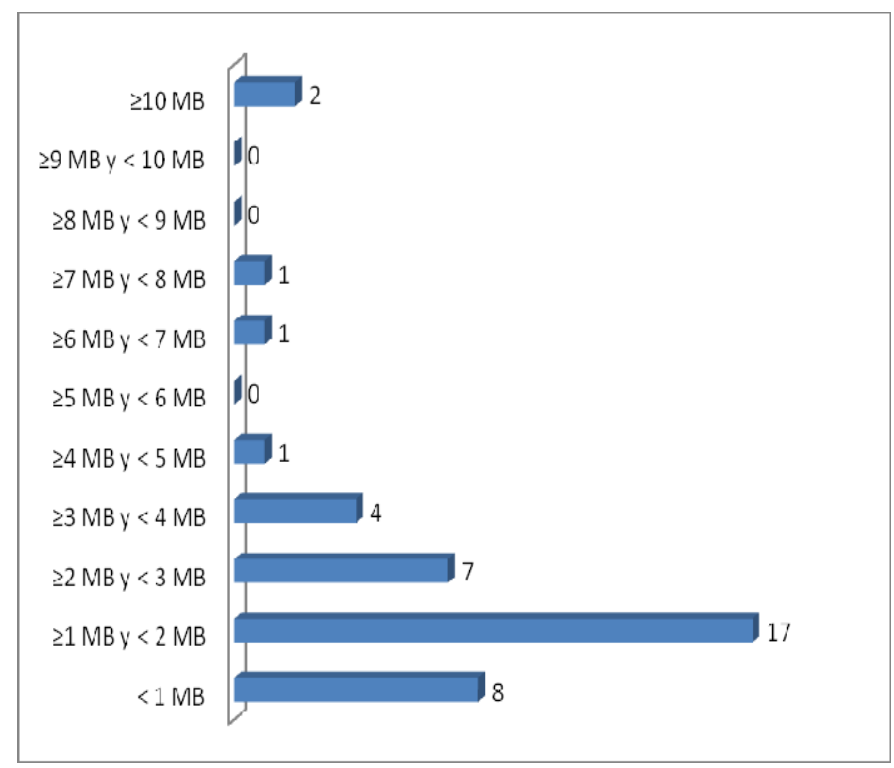

Fig. 11. Cantidad de Sitios Web

\section{CONCLUSIONES}

El gobierno electrónico puede ser favorecido por las TIC para mejorar la comunicación en el espacio G2C. Los nuevos canales deben sumarse a los ya existentes, la diversidad de canales darán más opciones al ciudadano al momento de necesitar buscar información o interactuar con los organismos públicos. Producto del presente artículo se puede advertir que los SMS han sido implementados a nivel mundial para brindar información y servicios a los ciudadanos, Argentina también tiene diversas implementaciones por este canal de comunicación. No obstante -en los resultados obtenidos del relevamiento de los 135 Municipios de La Provincia de Buenos Aires (incluyendo a la Ciudad Autónoma de Buenos Aires), lo que representa al $46 \%$ de la población en Argentina- aún hay un $7 \%$ de municipios que no tiene sitio web oficial. El problema principal radica en que los gobiernos locales hacen esfuerzos aislados para poder implementar las distintas soluciones tecnológicas con un presupuesto reducido para tal fin. La falta de esfuerzos mancomunados dificulta la integración de soluciones y la interoperabilidad de las mismas. 
El $96 \%$ de los municipios no cuenta con un sitio web móvil. No obstante cabe destacar que el 30\% han recurrido al diseño adaptativo para ofrecer sitios que se visualicen mejor en los dispositivos móviles, pero tan sólo se trata de una cuestión de visualización, sin optimizar contenidos. Por otra parte cabe destacar que el diseño adaptativo es una forma de visualizar al mismo sitio, es decir que los sitios web con diseño adaptativo continúan siendo la misma solución de escritorio con el mismo peso excesivo, cargadas de recursos gráficos, que requieren plugins para ser visualizadas...

A pesar de la importancia del Gobierno Electrónico la cual ha sido valorada desde hace una década con las resoluciones oficiales en Argentina, el proceso de implementación de las TIC en la Administración Pública a beneficio de los ciudadanos se ha visto rezagado. Un claro ejemplo de esto son los Gobiernos Locales, que queda en evidencia a través del relevamiento realizado en Municipios de la provincia de Buenos Aires.

Aún hay mucho camino por recorrer en cuanto a la implementación de tecnología pero también en cuanto a la evaluación de la calidad de las soluciones puestas a disposición. Si bien no es el objeto de este artículo, es importante destacar que también es necesaria una modernización de la Administración Pública en sus procesos internos, lo cual conlleva esfuerzos que no siempre son visibles por la ciudadanía. No solo se trata de una posible resistencia al cambio o a la adopción de las TIC sino también falta de presupuesto para destinar a este fin. Es necesario tomar conciencia de la importancia de ofrecer nuevos canales de comunicación para favorecer el espacio G2C y que estos esfuerzos no sean aislados, sino que las distintas reparticiones públicas puedan contribuir a la construcción de soluciones que puedan implementarse en distintas reparticiones. Algunos países cuentan con templates de sitios web, los cuales son facilitados a los gobiernos locales. De esta forma todos tienen un aspecto uniforme y normalizado. Así también existen repositorios de servicios web que pueden ser consumidos.

En Argentina no hay estándares en los que se basen los desarrollos, ni siquiera las URL de acceso a los sitios tienen un formato estándar. Es evidente que existen muchos esfuerzos aislados y desconocimiento de recursos existentes. A pesar de ello es necesario no sólo incorporar nuevos recursos, sino asegurar la calidad de las soluciones ya existentes, de hecho la Universidad Nacional de La Matanza ha brindado asesoría a municipios para la auditoria de sus soluciones web a través del GIDFIS (Grupo de Investigación, Desarrollo y Formación en Innovación de Software) y producto de ello, muchos municipios han mejorado sus soluciones web.

\section{REFERENCIAS}

[1] O. Cabrera, "Transformaciones sociales a través de la comunicación digital hacia una sociedad del conocimiento." Colección Académica de Ciencias Sociales 1.1 2014. Pp. 139-152.

https://revistas.upb.edu.co/index.php/cienciassociales/article/vie $\mathrm{w} / 2888$

[2] G. Sunkel, D. Trucco. "Las tecnologías digitales frente a los desafíos de una educación inclusiva en América Latina: Algunos casos de buenas prácticas". Santiago de Chile: Comisión Económica para América Latina y el Caribe (CEPAL). 2012 http://repositorio.cepal.org/handle/11362/21658R.

[3] R. Rodríguez, P. Vera, M., Martínez, L. Verbel de La Cruz, "Aprovechamiento del hardware de los dispositivos móviles para la construcción de nuevas aplicaciones". In XVI Workshop de Investigadores en Ciencias de la Computación. 2014 http://hdl.handle.net/10915/42655

[4] Esteves J., "Análisis del desarrollo del Gobierno Electrónico Municipal en España", Madrid, 2005 http://latienda.ie.edu/working_papers_economia/WPE05-32.pdf

[5] Estevez, Elsa, Pablo Fillottrani, and Tomasz Janowski. "From egovernment to seamless government." Proceedings of the Conference on Collaborative Electronic Commerce Technology and Research, CollECTeR Iberoamerica 2007, Cordoba, Argentina. Vol. 1. 2007.

[6] R de Armas Urquiza, A de Armas Suárez."Gobierno electrónico: fases, dimensiones y algunas consideraciones a tener en cuenta para su implementación." Contribuciones a las Ciencias Sociales2011-07. 2011.

http://www.eumed.net/rev/cccss/13/auas.htm

[7] R. Rodríguez, "Marco de Medición de calidad para gobierno electrónico." XIV Workshop de Investigadores en Ciencias de la Computación. 2012.

http://hdl.handle.net/10915/19513

[8] Pagani, María Laura, and Valería Pau. "NTIC y modernización en los municipios de la provincia de Buenos Aires: resultados de un estudio sobre sus portales web." 2013.

http://www.gobiernolocal.gob.ar/sites/default/files/websmunicipales-2013---final.pdf

[9] UNESCO. "Gobernabilidad Electrónica. Fortalecimiento de capacidades de gobernabilidad Electrónica". http://portal.unesco.org/ci/en/files/14896/11412266495egovernance.pdf/e-governance.pdf

[10] Concha, Gastón, and Alejandra Naser. "El desafío hacia el gobierno abierto en la hora de la igualdad." Comisión Económica para América Latina y el Caribe (CEPAL). 2012. http://iis7e2.cepal.org/ddpe/publicaciones/xml/9/46119/W465.pdf

[11] Ministerio de Economía y Finanzas Públicas. Presidencia de la Nación. InfoLEG (Información Legislativa y Documental). "Plan nacional de gobierno electronico y planes sectoriales de gobierno electrónico". Decreto 378/2005. Argentina. 2005.

http://infoleg.mecon.gov.ar/infolegInternet/anexos/105000109999/105829/norma.htm

[12] Ministerio de Economía y Finanzas Públicas. Presidencia de la Nación. InfoLEG (Información Legislativa y Documental). "Carta iberoamericana de gobierno electrónico". Chile. 2007. http://infoleg.mecon.gov.ar/infolegInternet/anexos/105000109999/105829/norma.htm

[13] CLAD. "Carta iberoamericana de Calidad en la Gestión Pública". El Salvador. Resolución $\mathrm{N}^{\circ} 25$ del "Plan de Acción de San Salvador". 2008.

http://observatorioserviciospublicos.gob.do/baselegal/carta_iber oamericana_de_calidad.pdf

[14] CLAD. "Carta iberoamericana de Gestión Pública". Resolución No 38 del Plan de Acción de Lisboa. 2009.

http://observatorioserviciospublicos.gob.do/baselegal/carta_iber oamericana_de_participaci\%C3\%B3n_ciudadana.pdf

[15] R. Rodríguez. "Gobierno en Línea, Análisis de la fase transaccional". II Encuentro Regional de Experiencias Exitosas: Gobierno en Línea Territorial, Colombia. 2012 https://youtu.be/9KbI6STWm3E

[16] Finquelievich, Susana, Patricio Feldman, and Celina Fischnaller. "Los territorios urbano-regionales como medio de innovación: San Luis, ¿laboratorio ciudadano?." Revista iberoamericana de ciencia tecnología y sociedad 8.23 (2014): 151-177.

[17] Romaní, J. C. C. El concepto de tecnologías de la información. Benchmarking sobre las definiciones de las TIC en la sociedad del conocimiento. Zer-Revista de Estudios de Comunicación, 14(27). 2011

http://www.ehu.eus/ojs/index.php/Zer/article/view/2636 
[18] I. Kushchu, H. Kuscu, From E-government to M-government: Facing the Inevitable. In the 3rd European Conference on eGovernment (pp. 253-260). MCIL Trinity College Dublin Ireland. 2003.

[19] R. Rodríguez, D. Giulianelli, A. Trigueros, P. Vera, I. Marko. "Los organismos gubernamentales y la estrategia de la utilización de mensajes de texto para ofrecer m-Servicios". 2010 http://hdl.handle.net/10915/19369

[20] United Nations. "E-government survey 2014 - E-government for the future we want", 2014.

http://unpan3.un.org/egovkb/Portals/egovkb/Documents/un/201 4-Survey/E-Gov_Complete_Survey-2014.pdf

[21] Arujo, Mentxu Ramilo. "Gobiernos, administraciones y ciudadanía en la red."Cuadernos de Gobierno y Administración Pública 1.1. 2014. pp 77-96.

http://revistas.ucm.es/index.php/CGAP/article/download/45159/ 42521

[22] Subsecretaría de Asuntos Municipales. Ministerio de Gobierno, Buenos Aires Provincia. "Municipios de la provincia de Buenos Aires". http://www.gobierno.gba.gov.ar/cdi/frames/central.html

[23] Dirección Provincial de Estadística. Ministerio Economía. "Censo 2010. Provincia de Buenos Aires. Resultados Definitivos por Partido". 2010 http://www.ec.gba.gov.ar/estadistica/librocenso2010.pdf

[24] INDEC. "Censo Nacional de Población, Hogares y Viviendas 2010”. Resultados Definitivos Serie B N 2 Tomo 1. 2010 http://www.censo2010.indec.gov.ar/archivos/censo2010_tomo1. pdf

[25] R. Rodríguez, L. Welicki, D. Giulianelli, P. Vera "Measurement framework for evaluating e-governance on municipalities websites."Proceedings of the 2 nd international conference on Theory and practice of electronic governance. ACM, 2008.

[26] J. Cuello; J. Vittone. "Diseñando Apps móviles". Primera edición: junio de 2013. Versión 1.1 ISBN (PDF): 978-84-6165070-5 ISBN (ePub): 978-84-616-4933-4

[27] W3C, Mobile Web Best Practices 1.0, 2008 http://www.w3.org/TR/mobile-bp/

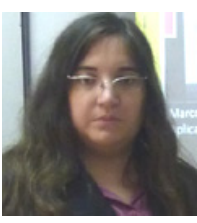

Rocío Andrea Rodríguez. Ingeniera en Informática (UNLaM-Universidad Nacional de La Matanza), Doctora en Ciencias Informáticas (UNLP-Universidad Nacional de La Plata). Es docente de Grado en la UNLaM y UTN (Universidad Tecnológica Nacional); docente de postgrado en la UAI (Universidad Abierta Interamericana) y UNLaM. Directora Académica del GIDFIS (Grupo de Investigación y Desarrollo en Innovación de Software). Directora de proyectos de investigación en la UAI y UNLaM. Además dirige pasantes, becarios y tesistas. Ha participado como jurado de tesis y revisora de: artículos, proyectos de extensión universitaria y programas cofinanciados. Siendo autora de: libros, capítulos de libros y artículos académicos.

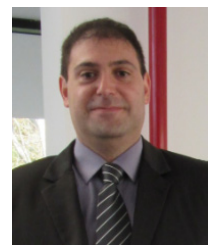

Pablo Martín Vera. Ingeniero en Informática (UNLaM-Universidad Nacional de La Matanza). Doctor en Ciencias Informáticas (UNLPUniversidad Nacional de La Plata). Docente de grado en el UNLaM y UTN (Universidad Tecnológica Nacional). Docente de posgrado en UNLaM y en la UAI (Universidad Abierta Interamericana). Co-director de proyectos de investigación en UNLaM y UAI. Supervisor de PPS (Prácticas Profesionales Supervisadas) de alumnos de Ingeniería en UNLaM, Director de Becarios (UAI y UNLaM). Revisor de publicaciones académicas. En el ámbito privado, es Director de Tecnología en una empresa de telecomunicaciones.

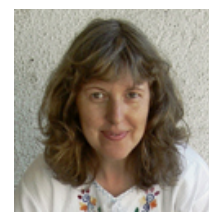

Isabel Beatriz Marko. Ingeniera en Electrónica (UBA- Universidad Buenos Aires), Master en Docencia Universitaria (UTN- Universidad Tecnológica Nacional Regional Buenos Aires). Docente de Grado en UNLaM (Universidad Nacional La Matanza) y UTN. Jefa de Cátedra de las materia Informática Avanzada y Diseño de Interfaces (UNLaM). Integrante del Consejo Superior de la Universidad J. F. Kennedy. Integrante del GIDFIS. Co-Directora de proyectos de investigación en la UNLaM. Es tutora de proyecto final de Ingeniería Electrónica (UNLaM). Ha presentado trabajos en eventos científicos (jornadas, congresos, seminarios, etc.). Trabaja en ámbito privado en productos y soluciones para CATV, Comunicaciones y Sistemas de Información.

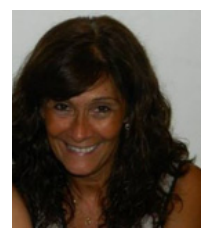

Claudia Gabriela Alderete. Licenciada en Sistemas (UM- Universidad de Morón). Es docente de Grado en UNLaM (Universidad Nacional de La Matanza). Es Tutora del Departamento de Ingeniería e Investigaciones Tecnológicas (DIIT) en UNLaM, para alumnos de Ingeniería que transitan el Ciclo General de Conocimientos Básicos. Integrante del GIDFIS (Grupo de Investigación Desarrollo y Formación en Innovación de Software - UNLaM). Es autora de diversas publicaciones académicas vinculadas con proyectos de Investigación y Desarrollo.

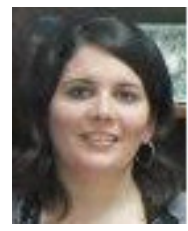

Anabella Graciela Conca. Profesora de Tercer Ciclo de EGB y de la Educación Polimodal en Matemática (Instituto Superior Padre Elizalde). Estudiante de Ingeniería en Informática (UNLaM Universidad Nacional de La Matanza) y de la Licenciatura en Matemática Aplicada (UNLaM Universidad Nacional de La Matanza). Docente en el nivel terciario (Instituto Superior Padre Elizalde), nivel secundario (Instituto Modelo Proyecto XXI). Se desempeña como ayudante alumno en el Departamento de Ingeniería e Investigaciones Tecnológicas de la UNLaM. También es miembro del GIDFIS (Grupo de Investigación y Desarrollo en Innovación de Software) en UNLaM. 